\title{
Assessment of Interleukin-12 Gene Polymorphism in Vitiligo Patients
}

H.H.Sabry ${ }^{1}$, N.F.Ibrahim ${ }^{2}$, A.I.Mustafa ${ }^{1}$ and A.A.Abdel-Monsef ${ }^{1}$

${ }^{1}$ Dermatology, Venereology \& Andrology Dept., Faculty of Medicine, Benha Univ., Benha, Egypt

${ }^{2}$ Biochemistry and Molecular Biology Dept., Faculty of Medicine, Benha Univ., Benha, Egypt

E-Mail:asmaa255@gmail.com

\begin{abstract}
Vitiligo is an obtained pigmentary turmoil of the skin and mucous films. Interleukin-12 has a positive synergistic proliferative impact on pre-initiated NK and T cells, improves freely and additionally synergistically the cytolytic capacity of both NK and CD8+ T cells by upregulation of qualities that encode cytotoxic cell granule-related proteins. The point of the work is to uncover the relationship between IL-12 quality polymorphism and vitiligo pathogenesis among contemplated gathering of Egyptian populace. This case-control study included 50 patients experiencing vitiligo (Group A) and 50 obviously solid people of coordinated age and sex as a benchmark (Group B). All patients were exposed to the followings: Full history taking, Clinical assessment, and Laboratory examinations for the genotypes of interleukin-12 (1188 A/C) SNP. Results and Conclusion: The IL12B rs3212227 CC genotype and C allele indicated fundamentally lower recurrence in situations when contrasted with control bunches with defensive impact to create vitiligo cases. Negative family ancestry, non-smokers and CC genotype were viewed as defensive indicators against vitiligo powerlessness. More seasoned period of beginning, more limited illness term, CA and CC genotypes were viewed as free indicators of less extreme vitiligo patients. By and by, bigger examinations are as yet expected to affirm our discoveries and to research the function of - 12 hereditary polymorphism in sickness seriousness.
\end{abstract}

Keywords: Interleukin-12, Gene Polymorphism, Vitiligo.

\section{Introduction}

Vitiligo is an obtained pigmentary confusion of the skin and mucous films that is described clinically by the advancement of all around surrounded white macules and patches of skin and hair with nonattendance of melanocytes minutely. Around the world, the commonness of vitiligo goes from 0.4 to $2.0 \%$, with districts of more noteworthy or lesser predominance. It might show up at whatever stage in life and influence both genders [1].

Vitiligo is classified into non-segmental and segmental subtypes; the last happen in a minority $(5-16 \%)$ of patients. Beginning and course of the illness may differ by subtype. What's more, people with vitiligo may encounter critical mental appearances [2].

The specific pathogenesis of vitiligo is still to be explained. Different components, including metabolic irregularities, oxidative pressure, age of fiery go betweens, cell separation and immune system reactions, may add to the pathogenesis. Other pathogenic causes may incorporate hereditary impacts, sensory system irregular characteristics. Specifically, the immune system component is plainly settled [3].

Vitiligo has likewise been related with thyroid issues, especially Hashimoto thyroiditis and Graves' sickness, different endocrinopathies, for example, Addison infection, type 1 diabetes mellitus, alopecia areata, poisonous iron deficiency, fiery inside illness, psoriasis, and immune system poly-glandular disorder are completely connected with vitiligo. In vitiligo, unmistakable highlights are apoptosis of melanocytes and keratinocytes brought about by cytotoxic resistant response, and breakdown of utilitarian melanocytekeratinocyte crosstalk needed for typical pigmentation. There is persuading proof that the harm and loss of melanocytes in vitiligo is interceded via immune system assault of CD8+ T cells. The trigger for this cell-interceded response is as yet unclear [4].
Interleukin 12 (IL-12), a favorable to fiery heterodimeric cytokine, is the primary individual from the IL-12 cytokine family, additionally including IL-23, IL-27 and IL-35. IL-12 is made out of p35 and p40 subunits otherwise called IL-12 An and IL-12B consecutively which when consolidated together structure the bioactive IL-12p70. IL12A is situated on chromosome 3 and IL12B is situated on Chromosome 5. IL-12 is mostly emitted from antigen introducing cells (phagocytes, dendritic cells) because of microbes, advancing CD4+ cell separation into Th1 cells which produce cytokines; IL-10, Interferon (IFN- $\gamma$ ). IFN- $\gamma$ may partake in the homing of CD8 $+\mathrm{T}$ cells to the skin through neighborhood enlistment of chemokines and articulation of grip particles on endothelial cells. Cytotoxicity by means of acceptance of apoptosis has been considered as a significant effector instrument of autoreactive CTLs. Hence, selected CD8+ T cells may specifically instigate autologous melanocyte apoptosis bringing about vanishing of melanocytes [5].

Then again, CD4+ Th1 cells (one of the principle wellsprings of IFN- $\gamma$ creation) are needed for ideal enlistment of antigen-specific CD8 $+\mathrm{T}$ cell effector reactions. CD8+ $\mathrm{T}$ cells can be considered as a principle effector cell of Th1 invulnerability. Thusly, participation somewhere in the range of $\mathrm{CD} 4+$ and $\mathrm{CD} 8+\mathrm{T}$ cells assumes a significant function in complete melanocyte leeway [6].

Interleukin-12 has a positive synergistic proliferative impact on pre-actuated NK and T cells, improves freely and additionally synergistically the cytolytic capacity of both NK and CD8+ T cells by upregulation of qualities that encode cytotoxic cell granule-related proteins [5].

The point of the work is to uncover the relationship between IL-12 quality polymorphism and vitiligo pathogenesis among contemplated gathering of Egyptian populace. 


\section{Patients and methods}

\subsection{Study Population}

This case-control study included 50 patients suffering from vitiligo (Group A) and 50 apparently healthy individuals of matched age and sex as a control group (Group B). Patients were recruited from the outpatient clinic of Dermatology and Andrology Department of Benha University Hospitals in the period between March and May 2019.

The study was approved by the local ethics committee on research involving human subjects of Benha Faculty of Medicine. An Informed consent was obtained from each individual before sample collection.

\subsection{Inclusion criteria}

All patients were enrolled in the study had:

- Clinically typical vitiligo lesions with different clinical varieties of vitiligo, and disease extent that was assessed by body surface area (BSA) \% affected.

\subsection{Exclusion criteria}

- Patients with vitiligo associated with systemic disease were excluded as; bronchial asthma and type1diabetes mellitus.

- Patients with segmental vitiligo.

- Patients with other diseases associated with IL-12 gene polymorphism as; multiple sclerosis, atopic dermatitis, bronchial asthma, rheumatoid arthritis, psoriasis vulgaris, inflammatory bowel disease, type 1 diabetes, crohn's disease hepatocellular carcinoma, nasopharyngeal cancer, cervical cancer, brain tumour, colorectal cancer, esophageal cancer, Hashimoto thyroiditis, and tuberculosis.

\section{All patients were subjected to the followings:}

- Full history taking

- Clinical examination:
- Laboratory investigations for the genotypes of interleukin-12 (1188 A/C) SNP.

\subsection{Statistical analysis}

The collected data was revised, coded and tabulated using Statistical package for Social Science (IBM Corp. Released 2017. IBM SPSS Statistics for Windows, Version 25.0. Armonk, NY: IBM Corp.). Data were presented and suitable analysis was done according to the type of data obtained for each parameter. Shapiro test was done to test the normality of data distribution. Significant data was considered to be nonparametric. Descriptive statistics: Mean, Standard deviation $( \pm$ SD) for numerical data. Frequency and percentage of non-numerical data. Analytical statistics: Student T Test was used to assess the statistical significance of the difference between two study group means. For the comparison of the three groups' means, one way analysis of variance (ANOVA) was used. Chi-Square test was used to examine the relationship between two qualitative variables. Fisher's exact test: was used to examine the relationship between two qualitative variables when the expected count is less than 5 in more than $20 \%$ of cells. Regression analysis: Logistic and linear regression analyses was used for prediction of risk factors. Odds ratio and $95 \%$ confidence interval were calculated. Deviations from Hardy-Weinberg equilibrium expectations were determined using the chi-squared test. All reported $p$ values were two-tailed and $p<0.05$ was considered to be significant.

\section{Results}

This study included 50 patients suffering from vitiligo. The mean age of studied cases was 30.8 years. They were 16 males (32\%) and 34 females (68\%). In addition, 50 apparently healthy individuals of matched age and sex was added as a control group ( $\mathrm{p}>0.05)$, each as shown in Table (1).

Table (1) Comparison of age and Sociodemographic distribution between all studied groups.

\begin{tabular}{|c|c|c|c|c|c|c|c|}
\hline & & & $\begin{array}{c}\text { Control } \\
\mathrm{N}=50\end{array}$ & & $\begin{array}{c}\text { Vitiligo } \\
\mathbf{N}=50\end{array}$ & & $\bar{p}$ \\
\hline Age & & mean \pm SD & 28.5 & 5.3 & 30.8 & 9.1 & 0.327 \\
\hline \multirow[t]{2}{*}{ Sex } & Male & $\mathrm{N}, \%$ & 21 & 42 & 16 & 32 & 0.300 \\
\hline & Female & $\mathrm{N}, \%$ & 29 & 58 & 34 & 68 & \\
\hline
\end{tabular}

The cases and control subjects were selected randomly from Al-Qalyubia Governorate in Egypt. They were unrelated. Applying Hardy Weinberg equation revealed that rs3212227 genotypes in control and cases groups were in equilibrium ( $\mathrm{p}>0.05)$, as shown in Table (2).

Table (2) Assessment of Hardy Weinberg equilibrium of studied SNP in vitiligo and control groups.

\begin{tabular}{lccccc}
\hline rs3212227 & \multicolumn{3}{c}{$\begin{array}{c}\text { Control } \\
\text { N=50 }\end{array}$} & \multicolumn{2}{c}{$\begin{array}{c}\text { Vitiligo } \\
\text { N=50 }\end{array}$} \\
\cline { 2 - 6 } & Observed & Expected & Observed & Expected \\
\hline AA & 23 & 20.5 & 30 & 30.4 \\
AC & 18 & & 23.0 & 18 & 17.2 \\
CC & 9 & & 6.5 & 2 & 2.4 \\
p & & 0.122 & & & 0.729 \\
\hline
\end{tabular}


Linear regression analysis was conducted for prediction of higher VASI score in studied vitiligo cases, using age, gender, BMI, FH, smoking, systemic disease, rs3212227 genotypes as covariates. Younger age of onset, longer disease duration, progressive course, were associated with higher BSA, while AC and CC genotypes were associated with lower BSA in univariable analysis.
Considering significant risk factors in univariable into multivariable analysis revealed that younger age of onset, longer duration were considered independent predictors of more severe vitiligo cases; while AC and CC genotypes were considered as good prognostic factors for less severe vitiligo patients Table (3).

Table (3) Regression analysis for prediction of higher BSA in studied vitiligo cases.

\begin{tabular}{|c|c|c|c|c|c|c|c|c|}
\hline \multirow{2}{*}{ Age } & \multicolumn{4}{|c|}{ Univariable } & \multicolumn{4}{|c|}{ Multivariable } \\
\hline & \multirow{2}{*}{$\begin{array}{c}\mathbf{p} \\
0.631\end{array}$} & \multirow{2}{*}{$\begin{array}{c}\mathbf{O R} \\
1.114\end{array}$} & \multicolumn{2}{|c|}{ 95\% CI } & \multirow[t]{2}{*}{$\mathbf{p}$} & \multirow[t]{2}{*}{ OR } & \multicolumn{2}{|c|}{ 95\% CI } \\
\hline & & & 0.717 & 1.731 & & & & \\
\hline Gender & 0.684 & 1.101 & 0.693 & 1.748 & & & & \\
\hline BMI $\left(\mathrm{kg} / \mathrm{m}^{2}\right)$ & 0.839 & 1.151 & 0.296 & 4.472 & & & & \\
\hline Family history & 0.275 & 1.363 & 0.782 & 2.377 & & & & \\
\hline $\begin{array}{l}\text { History of systemic } \\
\text { disease }\end{array}$ & 0.614 & 1.263 & 0.510 & 3.129 & & & & \\
\hline Age of onset (years) & $<0.001$ & 0.956 & 0.949 & 0.964 & 0.012 & 0.984 & 0.971 & 0.996 \\
\hline Duration (years) & $<0.001$ & 1.128 & 1.090 & 1.168 & 0.043 & 1.032 & 1.022 & 1.065 \\
\hline Course & 0.001 & 2.165 & 1.351 & 3.467 & 0.841 & 1.029 & 0.777 & 1.362 \\
\hline rs3212227 & - & 1 & & & - & 1 & & \\
\hline $\mathrm{AC}$ & $<0.001$ & 0.238 & 0.183 & 0.308 & 0.001 & 0.476 & 0.311 & 0.730 \\
\hline $\mathrm{CC}$ & $<0.001$ & 0.118 & 0.063 & 0.222 & 0.002 & 0.305 & 0.144 & 0.642 \\
\hline
\end{tabular}

OR, odds ratio; CI, confidence interval. Logistic regression test was used.

\section{Discussion}

Despite the fact that it can begin at whatever stage in life, vitiligo frequently first shows up between the ages of 20 and 40[7]. In the current examination, the mean age at the beginning of the infection was $23.3 \pm 7.2$ years.

In accordance with our discoveries, Mahajan and partners [8] examined clinico-epidemiological highlights of 945 patients with vitiligo with a goal to outline epidemiological and clinical parts of vitiligo from this piece of the nation. The clinical records of patients with vitiligo going to outpatient facility over a 5-year time frame from January 2013 to December 2017 were broke down reflectively for this expressive, observational investigation. The mean of the included patients was 24.4 years.

Also, Alissa and associates [9] evaluated the clinical examples, epidemiological profile of vitiligo, and its mindfulness among Saudi nationals. A review study was directed among Saudi nationals with an affirmed analysis of vitiligo introducing to the National Center for Vitiligo and Psoriasis, Riyadh, Saudi Arabia, from August 2002 to August 2006 utilizing a review poll dependent on the set of experiences and clinical records of patients. Of the 4134 cases, $53.5 \%$ were females. The mean age was 27.4 years.

The two people are in danger for the vitiligo, yet the rates might be marginally higher among females[10].

In the current investigation, most of patients were females $(68 \%)$.

Additionally, Paradisi and associates [11] looked to consider the overall danger of melanoma and in patients with vitiligo. In this gathering, 5457 were female (54.4\%) and 4583 were male $(45.6 \%)$.
Moreover, Alkhateeb and partners [12] reviewed 2624 probands in North America and the UK with respect to their vitiligo. They venture a populace based predominance of $0.4 \%$ for vitiligo in the joined districts, with an extended female pervasiveness of $0.41 \%$ and male commonness of $0.39 \%$. In this arrangement, $72 \%$ of the patients were female,

Family ancestry is one of the fundamental danger factors for vitiligo, given the immune system nature of the infection. It seems to lie somewhere in the range of 15 and $20 \%$ in many investigations [13]. In the current investigation, we noticed that practically $18 \%$ of the patients had positive family background of vitiligo.

In concurrence with our discoveries, Nejad and partners [14] examined the relationship among vitiligo and immune system infections. For this situation control study, 86 patients with vitiligo were incorporated. Normal time of sickness beginning was $21.8 \pm 11$ years; $61 \%$ of patients were female and $39 \%$ were male. Almost one-fourth of patients had a positive family background of vitiligo.

Sun and partners [15] investigated the conceivable hereditary model of vitiligo by breaking down the hereditary attributes of 815 patients and their families from south China (Zhejiang Province). Information for 815 patients with vitiligo were acquired by survey. In 815 vitiligo probands, $128(15.7 \%)$ had a family ancestry

Vitiligo can be ordered by the degree of color misfortune into nearby, summed up, or general subtypes. Nonsegmental vitiligo (otherwise called two-sided vitiligo, vitiligo vulgaris, and summed up vitiligo) is the most wellknown sort of vitiligo and results in white patches showing up on the two sides of the body[16]. 
In the current investigation, by far most of the patients had central vitiligo $(58 \%)$, trailed by summed up $(36.6 \%)$ and acrofacial $(6 \%)$ types. The sickness was reformist in $76 \%$ of the patients.

In spite of our discoveries, Esfandiarpour and Farajzadeh [17] broke down the epidemiological and clinical components of patients with late-beginning vitiligo and contrast these with patients with beginning stage vitiligo. 800 and 25 back to back patients with vitiligo were inspected from March 2003 through May 2006. The patients were partitioned into late-beginning (sickness beginning > 50 years old) and beginning stage gatherings. Summed up (vitiligo vulgaris) was the most widely recognized sort in the two gatherings.

Likewise, in their examination on 182 patients with late-beginning vitiligo, Dogra and associates [18] announced vitiligo vulgaris as the most widely recognized clinical sort followed by central subtype.

The specific reasons for such heterogeneity between our outcomes and the distributed writing are hazy. Notwithstanding, it tends to be credited to the little example size and review inclination (i.e relying upon patients' memory to address the historical backdrop of stressors).

The function of penetration of incendiary middle people is settled as significant patron for the reformist, immune system intervened, decimation of melanocytes seen in vitiligo[19]. On account of the pleiotropic exercises of IL-12, many atomic epidemiologic investigations have investigated the impacts of IL-12 polymorphisms on vulnerability of immune system infections. The most normally interesting loci were rs3212227 in IL-12B quality, rs568408 and rs2243115 in IL-12A qualities, maybe inferable from their capacity to impact IL-12 quality articulation, decrease protein union, and in this way bring about immune system infection [20].

Concerning the essential result of the current investigation, we found that the patients with vitiligo had similar pace of rs3212227 genotypes to ordinary populace. Taking rs3212227 AA as the reference genotype and An as the reference allele; $\mathrm{CC}$ genotype and $\mathrm{C}$ allele demonstrated essentially lower recurrence in situations when contrasted with control gatherings $(p=0.023,0.029$ separately), with defensive impact against vitiligo weakness $(\mathrm{OR}=0.341,95 \% \mathrm{CI}=0.135-0.863$; $\mathrm{OR}=0.650$, $\mathrm{OR}=0.441-0.958$ individually).

Apparently, this is the primary investigation which demonstrated a huge relationship between IL-12 hereditary polymorphism and vitiligo helplessness. Despite the fact that the specific systems by. Past reports exhibited that the degree of serum IL-12 is higher in vitiligo patients; for instance, Gholijani and partners [21] meant to discover the cytokine profile of intrinsic and versatile insusceptibility in vitiligo patients, and associate them with clinical boundaries. The serum levels of IL-12 of every 44 vitiligo patients were estimated by multiplex cytokine measure and contrasted and 44 solid subjects. The degree of serum IL-12 was higher in vitiligo patients.

Outstandingly, our outcomes indicated that IL-12 hereditary polymorphism was altogether connected with qualities of sickness seriousness, for example, body surface zone (BSA), infection length, and recurrence of reformist illness.

Once more, apparently, this is the main investigation which demonstrated a critical relationship between IL-12 hereditary polymorphism and vitiligo seriousness.

Our discoveries can be clarified by the way that IL-12prompted separation of Th1 cells may be related with the movement of organ-explicit immune system illnesses [22].

Regardless of whether the IL-12 hereditary polymorphism has an effect in patients with vitiligo stays uncertain. We recognize that the current examination has various constraints. The patients were not followed up for long haul to research the adjustment in serum IL-12 over the span of infections. Another restriction is the little example size which may have influence the generalizability of our discoveries.

\section{Conclusion}

Taking everything into account, the momentum study recommends a potential inclusion of IL-12 hereditary polymorphism in the advancement of vitiligo sores and it very well may be considered as a potential marker for screening of early illness. We found that the IL12B rs3212227 CC genotype and C allele had essentially lower frequencies in situations when contrasted with control bunches with defensive impact to create vitiligo cases. Outstandingly, our outcomes indicated that IL-12 hereditary polymorphism was fundamentally connected with qualities of sickness seriousness, for example, body surface territory (BSA), illness length, and recurrence of reformist infection. Additionally, - 12 hereditary polymorphism might be considered as a potential early pointer for appraisal of the achievement of the treatment. In any case, bigger examinations are as yet expected to affirm our discoveries and to research the part of - 12 hereditary polymorphism in illness seriousness

\section{References}

[1] A. Taïeb, M. Picardo, and other V. members, "The definition and assessment of vitiligo: a consensus report of the Vitiligo European Task Force," Pigment Cell Res., Vol. 20,PP.27-35, 2007.

[2] K. Ezzedine, V. Eleftheriadou, M. Whitton, and N. van Geel, "Vitiligo," Lancet, Vol. 386,PP.74-84, 2015.

[3] M. Picardo, "Pathophysiology overview," in Vitiligo, Springer, Vol. 2019, no. 3,PP.189-192, 2019.

[4] V. M. Sheth, Y. Guo, and A. A. Qureshi, "Comorbidities associated with vitiligo: a ten-year retrospective study," Dermatology, Vol. 227,PP.311315, 2013.

[5] M. J. Smyth, M. Taniguchi, and S. E. A. Street, "The anti-tumor activity of IL-12: mechanisms of innate immunity that are model and dose dependent," $J$. Immunol., Vol. 165,PP.2665-2670, 2000.

[6] S. K. Bromley, T. R. Mempel, and A. D. Luster, "Orchestrating the orchestrators: chemokines in control of T cell traffic," Nat. Immunol., Vol. 9,PP.970-980, 2008. 
[7] P. Manga, N. Elbuluk, and S. J. Orlow, "Recent advances in understanding vitiligo.," F1000Research, Vol. 5,PP.78-90, 2016.

[8] N. Mahajan, P. Arora, and R. Sandhir, "Perturbed Biochemical Pathways and Associated Oxidative Stress Lead to Vascular Dysfunctions in Diabetic Retinopathy," Oxid. Med. Cell. Longev., Vol. 2019,PP.54-78, 2019.

[9] A. Alissa, A. Al Eisa, R. Huma, and S. Mulekar, "Vitiligo-epidemiological study of 4134 patients at the National Center for Vitiligo and Psoriasis in Central Saudi Arabia.," Saudi Med. J., Vol. 32,PP.1291-6, 2011.

[10] A. B. Dillon, A. Sideris, A. Hadi, and N. Elbuluk, "Advances in Vitiligo: An Update on Medical and Surgical Treatments.," J. Clin. Aesthet. Dermatol., Vol. 10,PP.15-28, 2017.

[11] A. Paradisi, S. Tabolli, B. Didona, L. Sobrino, N. Russo, and D. Abeni, "Markedly reduced incidence of melanoma and nonmelanoma skin cancer in a nonconcurrent cohort of 10,040 patients with vitiligo," J. Am. Acad. Dermatol., Vol. 71,PP.1110-1116, 2014.

[12] A. Alkhateeb, P. R. Fain, A. Thody, D. C. Bennett, and R. A. Spritz, "Epidemiology of vitiligo and associated autoimmune diseases in Caucasian probands and their families.," Pigment cell Res., Vol. 16, no. 3,PP.208-14, 2003.

[13]N. B. Silverberg, "The Epidemiology of Vitiligo," Curr. Dermatol. Rep., Vol. 4,PP.36-43, 2015.

[14] S. B. Nejad, H. H. Qadim, L. Nazeman, R. Fadaii, and M. Goldust, "Frequency of autoimmune diseases in those suffering from vitiligo in comparison with normal population.," Pakistan J. Biol. Sci. PJBS, Vol. 16, no. 12,PP.570-4, 2013.

[15]X. Sun et al., "Genetic epidemiology of vitiligo: a study of 815 probands and their families from south China," Int. J. Dermatol., Vol. 45,PP.1176-1181, 2006

[16] A. Bishnoi and D. Parsad, "Clinical and Molecular Aspects of Vitiligo Treatments.," Int. J. Mol. Sci., Vol. 19,PP.243, 2018.

[17]I. Esfandiarpour and S. Farajzadeh, "Clinical characteristics of late-onset vitiligo in an Iranian population," Dermatologica Sin., Vol. 30,PP.43-46, 2012.

[18] S. Dogra, D. Parsad, S. Handa, and A. J. Kanwar, "Late onset vitiligo: A study of 182 patients," Int. J. Dermatol., Vol. 44,PP.193-196, 2005.

[19] M. Rodrigues, K. Ezzedine, I. Hamzavi, A. G. Pandya, and J. E. Harris, "New discoveries in the pathogenesis and classification of vitiligo," J. Am. Acad. Dermatol., Vol. 77,PP.1-13, 2017.

[20] Y. Zheng et al., "Role of interleukin-12 gene polymorphisms in the onset risk of cancer: A metaanalysis," Oncotarget, Vol. 8,PP.29795-29807, 2017.

[21] N. Gholijani, M. R. Yazdani, and L. Dastgheib, "Predominant role of innate pro-inflammatory cytokines in vitiligo disease," Arch. Dermatol. Res., Vol. 312,PP.123-131, 2020.

[22] L. Sun, C. He, L. Nair, J. Yeung, and C. E. Egwuagu, "Interleukin 12 (IL-12) family cytokines: Role in immune pathogenesis and treatment of CNS autoimmune disease," Cytokine, Vol. 75,pp.249-255, 2015. 